\title{
金届玻璃 $\left(\mathrm{Fe}_{1-x} \mathrm{Co}_{x}\right)_{78} \mathrm{Si}_{10} \mathrm{~B}_{12}$ 的 磁化感生各向异性随温度的变化
}

\author{
陈 笉 行 \\ (钢铁研究总院, 北京)
}

\section{一、引}

对于金属玻璃的磁感生各向异性常数 $K_{u}$ 随温度的变化, 已经发现有如下关系成立:

$$
K_{u}(T)-k M_{s}^{a}(T),
$$

其中 $K_{\star}(T)$ 和 $M_{0}(T)$ 为温度 $T$ 下的磁感生各向异性常数和饱和磁化强度, $k$ 和 $\alpha$ 为常数. 现有的工作曾得到不同的 $\alpha$ 值. 对于 FeNiPB 的磁化感生各向异性, Egami 等和 Allia 等 得到 $\alpha-2$, Luborsky ${ }^{[3]}$ 得到 $\alpha=3.7$. 对于几种近零磁致伸缩 $\mathrm{Co}$ 基合金的磁化感生和应 变感生各向异性, Flanders 等 ${ }^{[4]}$ 和作者 ${ }^{[3}$ 分别得到 $\alpha-2.6$ 和 3. 对于 ( $\left.\mathrm{Fe}_{1-x} \mathrm{Co}_{x}\right)_{78} \mathrm{Si}_{10} \mathrm{~B}_{12}$ 的指 定温度横磁或横磁张力退火后的感生各向异性,作者得到 $\boldsymbol{\alpha}$ 值随 $\boldsymbol{x}$ 而异, 处于 3.3 至7.5之间 ${ }^{[6]}$. 按照磁性原子对的方向有序理论，感生各向异性常数 $K_{w}$ 随温度的变化应符合式 (1), 并且 $\alpha=2^{[7]}$. 但是,最近发现金属玻璃 $\mathrm{Fe}_{39} \mathrm{CO}_{39} \mathrm{Si}_{10} \mathrm{~B}_{12}$ 在阶跃温度退火时, $K_{u}$ 表现出明显的 Crossover 效应, 同时伴之以 $\alpha$ 值的突变. 这一现象不能用一般的方向有序理论解释, 而应与金属 玻琌中局域居里点的极宽分布有关 ${ }^{[8]}$. 根据这一观点，金属玻璃的 $\alpha$ 值随退火工艺而变应是 普遍现象. 为证实这一设想, 本工作对几种 $\left(\mathrm{Fe}_{1-x} \mathrm{CO}_{x}\right)_{78} \mathrm{Si}_{10} \mathrm{~B}_{12}$ 合金，在不同温度下进行横磁 退火, 研究磁化感生各向异性常数 $K_{\text {m }}$ 随温度的变化.

\section{二、样品和实验方法}

成分为 $\left(\mathrm{Fe}_{1-x} \mathrm{Co}_{x}\right)_{78} \mathrm{Si}_{10} \mathrm{~B}_{12}(x-0.7,0.9$ 和 1$)$ 的金属玻璃窄带用单辊快淬法制备. $\mathrm{X}$ 光 行射实验证实所得合金带是非晶态的. 示差扫描热分析测定 (升温速率 $10^{\circ} \mathrm{C} / \mathrm{min}$ ), 三种合金 的开始晶化温度 $T_{\mathrm{cry}}$ 分别为 440,465 和 $420^{\circ} \mathrm{C}$. 磁热天平在 $800 \mathrm{kA} / \mathrm{m}$ 磁场 $10^{\circ} \mathrm{C} / \mathrm{min}$ 升温 速率下所测的磁矩-温度关系表明, $x=0.7$ 合金的居里点 $T_{\mathrm{c}}$ 远高于 $T_{\mathrm{cr}}, x=0.9$ 和 1 合金 的 $T_{\mathrm{c}}$ 约为 515 和 $460^{\circ} \mathrm{C}$.

每种合金取一根长约 $45 \mathrm{~cm}$ 的样品, 在加热区长 $20 \mathrm{~cm}$ 的石英管卧式无感电热丝炉中进 行退火温度 $T_{\mathrm{a}}$ 依次提高的横磁退火. $T_{2} \leqslant 450^{\circ} \mathrm{C}$ 时保温 $20 \mathrm{~min}, T_{\mathrm{a}}=500^{\circ} \mathrm{C}$ 时保温 $10 \mathrm{~min}$ $(x-0.7)$ 或 $5 \min (x-0.9)$. 每次退火时在 $2 \min$ 内升到 $T_{a}$, 而降温初始冷速为 $150^{\circ} \mathrm{C} / \mathrm{min}$. 退火自始至终用电磁铁施加样品带面横向的磁场 $100 \mathrm{kA} / \mathrm{m}$. 为测量磁性, 炉管外套有水冷螺 线管以施加纵向磁化场, 样品带上套有 $6 \mathrm{~cm}$ 长探测线圈, 在测量前滑到样品加热区中段, 仍用 石英管炉加热。在从室温到 $200^{\circ} \mathrm{C}$ 的几个温度 $T$ 下保温, 用零位冲击反向法测量 $8 \mathrm{kA} / \mathrm{m}$ 磁场下 的磁化强度 $M_{8}$ 和磁化强度 $M=M_{8} / 2$ 点的磁化率 $\chi$, 按下式计算磁化感生各向异性常数 $K_{u}$ ： 本文1984 年5月21日收到. 


$$
K_{u}=\mu_{0} M_{\mathrm{B}}^{2} / 2 \chi
$$

因为 $8 \mathrm{kA} / \mathrm{m}$ 已接近饱和磁场,所以可将 $M_{8}$ 视为 $M_{s 。}$ 并在横磁退火后,易轴在横向的感生各 向异性具有压倒优势，磁化曲线接近直线，用可逆畴转磁化率 $\chi$ 计算 $K_{w}$ 的办法是可行的 ${ }^{(6)}$.

\section{三、实验结果}

图 1 给出由 $300-450^{\circ} \mathrm{C}$ 退火后的 $x-0.9$ 样品的测量结果算出的 $\lg K_{m}$ 随 $\lg \mu_{0} M$ ，的 变化. 可以看到 $\lg K_{m}$ 与 $\lg \mu_{0} M$ s 呈直线关系, 由直线的斜率得出 $T_{2}-300,350,400$ 和 $450^{\circ} \mathrm{C}$ 时 $\alpha$ 分别为 $5,4.5,4.0$ 和 3.1. 对于 $T_{2}-500^{\circ} \mathrm{C}\left(>T_{\mathrm{cr}}\right)$ 的退火, 得不到这种直线关 系, 故图中末给出. $x=0.7$ 的样品在稍高于 $T_{\mathrm{crg}}$ 的 $450^{\circ} \mathrm{C}$ 退火后, 虽然由于巴克豪森效应, 测量磁性的重复性较差, 但仍能基本上保持 $\lg K_{k}$ 与 $\lg \mu_{0} M_{s}$ 间的直线关系, 故仍可得出 $\alpha$ 值.

图 2 给出三种合金的 $K_{w}$ 和 $\alpha$ 随 $T_{\mathrm{a}}$ 的变化. 图中 $Q$ 表示淬态, 其 $K_{w}$ 为按式(2)得出的有 效各向异性常数,可以看到退火后的 $K_{u}$ 均比它们大得多. 虚线右边的实验点已显示了晶化的 作用,图 $2 \mathrm{~b}$ 和 $\mathrm{c}$ 中虚线所指的数字为 500 和 $450^{\circ} \mathrm{C}$ 退火后以非晶态的 $M$, 代入式 (2) 计算的 $K_{u}$ 。 中间以实线相连的几点应是非晶态的磁化感生各向异性常数. 三种合金的 $K_{u}$ 随 $T_{2}$ 的 变化情况不同: $x=0.7$ 者 $K_{u}$ 在 $T_{\mathrm{a}}<350^{\circ} \mathrm{C}$ 陡升后稍降, $x=1$ 者 $K_{u}$ 随 $T_{\mathrm{a}}$ 稳步上升, $x=$ 0.9 者居于二者之间. 三种合金的 $\alpha$ 随 $T_{2}$ 提高分别从 6.2, 5.0 和 7.1 单调下降至 3.0, 3.0 和 4.8 .
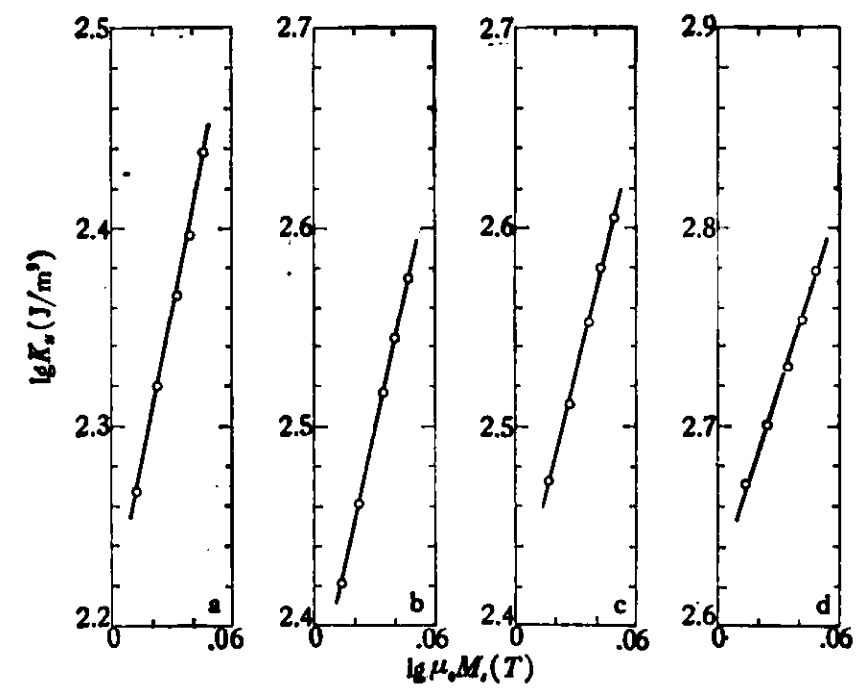

图 $1 x=0.9$ 样品在 $300(\mathrm{a}) 、 350(\mathrm{~b}) 、 400(\mathrm{c})$ 和 $450(d){ }^{\circ} \mathrm{C}$ 退火后的 $\lg K_{*}$ 随 $\lg \mu_{0} M$ ，的变化

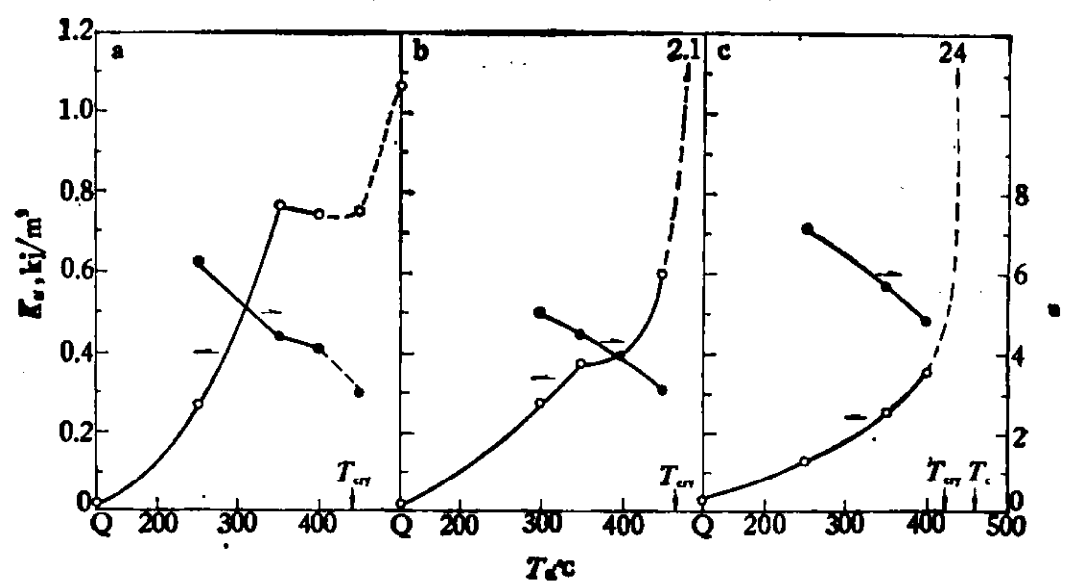

图 $2 x=0.7$ (a)、0.9(b) 和 1(c) 合金的 $K_{\mathrm{u}}$ 和 $\alpha$ 随 $T$ 的变化 


\section{四、讨 论}

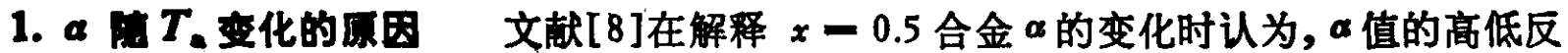
映 $K_{\mathrm{w}}$ 所涉及的原子团的平均居里点 $T_{\mathrm{c}}^{\prime}$ 的高低. 这种解释对本实验也是适用的. 在淬态样品 中含有较多自由体积，低温磁退火时自由体积圆周围的原子团具有较低的激活能而首先方向 有序化, 同时这些较松动的原子间交换作用也较弱,因而 $T_{\mathrm{c}}^{\prime}$ 也较低, 所以 $\alpha$ 较高. $T_{\mathrm{a}}$ 提高后， 结构驰豫使自由体积减少,方向有序化的原子团的 $T_{c}^{\prime}$ 提高,使 $\boldsymbol{\alpha}$ 下降.

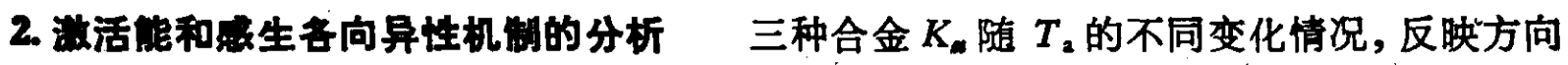
有序原子团激活能的分布不同. $x=0.7$ 合金 $K_{w}$ 在 250 到 $350^{\circ} \mathrm{C}$ 之间急开，说明在此温区有 大量原子团被激活，因而可以说激活能分布较窄。如果取弛豫时间 $\tau$ 为退火时间 $1200 \mathrm{~s}$, 并且 取通常的 $\tau_{0}$ 值 $10^{-13} s$ ，则由 Arrhenius 方程可得激活能为

$$
\Delta E=N k T_{2} \ln \left(\tau / \tau_{0}\right)=308 T_{a},
$$

式中 $N$ 为阿伏伽德罗数, $k$ 为玻耳兹曼常数, $T_{2}$ 的单位为 $\mathrm{K}, \Delta E$ 的单位为 $\mathrm{J} / \mathrm{mol}$. 因为 $K_{*}$ 急升 发生在 $T_{\mathrm{a}}=523$ 和 $623 \mathrm{~K}$ 之间, 将其中值 $573 \mathrm{~K}$ 代人式(3)是合适的, 这样得出的平均激活能约 $180 \mathrm{~kJ} / \mathrm{mol}(1.8 \mathrm{eV}) . x=1$ 合金的 $K_{w}$ 在 250 到 $400^{\circ} \mathrm{C}$ 稳步上升, 说明激活能较均匀的分布, 用式 （3）计算的 $\Delta E=150-210 \mathrm{~kJ} / \mathrm{m}^{3}$. $x=0.9$ 合金的情况居于 $x=0.7$ 和 1 之间. 注意到 $x=1$ 合金 中只有一种磁性原子 $\mathrm{Co}$ 而 $x=0.7,0.9$ 合金中则有二种磁性原子 $\mathrm{Co}$ 和 $\mathrm{Fe}$, 以上方向有序动力 学特点的差别可能反映了方向有序机制的差别. 对于 $x=0.7$ 者, 同类原子对的方向有序应该 起主导作用,而原子对的转向步及较集中的激活能. 对于 $x-1$ 者, 畸变原子团 ${ }^{[9]}$ 的方向有序 可能是感生各向异性的主要来源, 这些原子团的转向难易差别较大, 海及较宽的激活能分布.

3. 工化的作用 在 $T_{\mathrm{a}}$ 接近或高于 $T_{\mathrm{cry}}$ 时, 将发生晶化. 晶化使各向异性增大是由于 高矫顽力微晶的析出,它一方面通过强烈的静磁耦合阻碍母体的反磁化，一方面对母体施加高 的弹性应力, 增大应力各向异性. 从图 2 中三种合金晶化后 $K_{n}$ 的比较可以看出, Co 含量愈 高 $K_{w}$ 合大. 显然, 这反映了微晶的磁晶各向异性之差别. 文献 [10]指出, 本合金系的初期晶 化相为 $\mathrm{Fe}_{1-x} \mathrm{CO}_{x}$ 的室温平衡相. 六方 $\mathrm{Co}$ 和体心立方 $\mathrm{Fe}_{0.3} \mathrm{Co}_{0.7}$ 的磁晶各向 异性常数 $K_{1}$ 分 别为 460 和 $-42 \mathrm{~kJ} / \mathrm{m}^{3[12]}$, 所以 $x-1$ 合金在晶化后将有较 $x-0.7$ 合金大得多的 $K_{*}$.

\section{五、小结}

金属玻㻦 $\left(\mathrm{Fe}_{1-x} \mathrm{Co}_{x}\right)_{78} \mathrm{~S}_{10} \mathrm{~B}_{12}\left(x=0.7,0.9\right.$ 和 1) 在逐渐提高的温度 $T_{2}$ 下进行 磁场退火, 所得到的感生各向异性 $K_{w}$ 随温度 $T$ 的变化符合 $K_{w}(T)=k M_{s}^{a}(T)$ 关系, 指数 $\alpha$ 随 $T_{\mathrm{a}}$ 的提 高而从 5.0-7.1 下降到 3-4.8. 这种情况可以用局域居里点和激活能的较宽分布解释.

[ 1 ] Egami, T., Flanders, P. J., AIP Conf. Proc., 29(1976), 220.

[ 2 ] Allia, P., Soardo, G. P., Vinai, F., Solid State Commun., 24(1977), 517.

[ 3 ] Luborsky, F. E., AIP Conf. Proc., 29(1976), 209.

[ 4 ] Flanders, P. J., Morito, N., J. Appl. Phys., 55(1984), 1778.

[5]陈笉行,钢铁研究总院学报, 4(1984), 1: 85.

[6]陈等行,物理学报, 33(1984).

[ 7 ] Slonczewski, I. $\Gamma$. in Magnetism, Vol. 1 (Eds. G. T. Rado, H. Suhl), Academic Press, New Lork, $1963,229$.

[ 8 ] 陈笉行,科学通报, 29(1984), 19:953-956.

[ 9 ] Egami, T., in Amorphous Metallic Alloys (Ed. F. E. Luborsky), Butterworths, London (preprint).

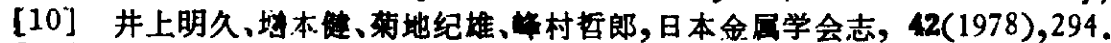

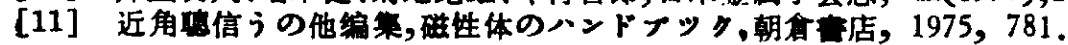

